\section{(C) OPEN ACCESS}

\title{
Relation between Chlamydia trachomatis infection and pelvic inflammatory disease, ectopic pregnancy and tubal factor infertility in a Dutch cohort of women previously tested for chlamydia in a chlamydia screening trial
}

\author{
Bernice M Hoenderboom, ${ }^{1,2}$ Birgit H B van Benthem, ${ }^{1}$ Jan E A M van Bergen, ${ }^{1,3,4}$ \\ Nicole H T M Dukers-Muijrers, ${ }^{5,6}$ Hannelore M Götz, 1,7,8 Christian J P A Hoebe, 5,6 \\ Arjan A Hogewoning, ${ }^{9}$ Jolande A Land ${ }^{10}$ Marianne A B van der Sande, ${ }^{1,11,12}$ \\ Servaas A Morré, ${ }^{2,10}$ Ingrid V F van den Broek ${ }^{1}$
}

\begin{abstract}
- Additional material is published online only. To view please visit the journal online (http://dx.doi.org/10.1136/ sextrans-2018-053778).
\end{abstract}

For numbered affiliations see end of article.

Correspondence to Dr Bernice M Hoenderboom, Centre for Infectious Disease Control (Clb) , National Institute for Public Health and the Environment (RIVM), Bilthoven 3720, The Netherlands; bernice. hoenderboom@rivm.nl

Received 25 July 2018 Revised 30 October 2018 Accepted 15 November 2018 Published Online First 3 January 2019

\section{Check for updates}

(C) Author(s) (or their employer(s)) 2019. Re-use permitted under CC BY-NC. No commercial re-use. See rights and permissions. Published by BMJ.

To cite: Hoenderboom BM, van Benthem BHB, van Bergen JEAM, et al. Sex Transm Infect 2019:95:300-306.

\section{ABSTRACT}

Objectives A better understanding of Chlamydia trachomatis infection (chlamydia)-related sequelae can provide a framework for effective chlamydia control strategies. The objective of this study was to estimate risks and risk factors of pelvic inflammatory disease (PID) ectopic pregnancy and tubal factor infertility (TFI) with a follow-up time of up until 8 years in women previously tested for chlamydia in the Chlamydia Screening Implementation study (CSI) and participating in the Netherlands Chlamydia Cohort Study (NECCST).

Methods Women who participated in the CSI 2008-2011 ( $n=13498$ ) were invited in 2015-2016 for NECCST. Chlamydia positive was defined as a positive CSI-PCR test, positive chlamydia serology and/or selfreported infection (time dependent). Data on PID, ectopic pregnancy and TFI were collected by self-completed questionnaires. Incidence rates and HRs were compared between chlamydia-positive and chlamydia-negative women corrected for confounders.

Results Of 5704 women included, 29.5\% (95\% Cl 28.3 to 30.7$)$ were chlamydia positive. The incidence rate of PID was 1.8 per 1000 person-years (py) (1.6 to 2.2) overall, 4.4 per 1000 py (3.3 to 5.7) among chlamydia positives compared with 1.4 per 1000 py (1.1 to 1.7) for chlamydia negatives. For TFI, this was 0.4 per 1000 py (0.3 to 0.5$)$ overall, 1.3 per 1000 py (0.8 to 2.1$)$ and 0.2 per 1000 py (0.1 to 0.4 ) among chlamydia positives and negatives, respectively. And for ectopic pregnancy, this was 0.6 per 1000 py (0.5 to 0.8$)$ overall, 0.8 per 1000 py (0.4 to 1.5 ) and 0.6 per 1000 py (0.4 to 0.8 ) for chlamydia negatives. Among chlamydia-positive women, the strongest risk factor for PID was symptomatic versus asymptomatic infection (adjusted HR 2.88, 1.4 to 4.5) and for TFI age $<20$ versus $>24$ years at first infection (HR 4.35, 1.1 to 16.8).

Conclusion We found a considerably higher risk for PID and TFI in chlamydia-positive women, but the incidence for ectopic pregnancy was comparable between chlamydia-positive and chlamydia-negative women. Overall, the incidence rates of sequelae remained low. Trial registration NTR-5597.

\section{BACKGROUND}

Chlamydia trachomatis infection (chlamydia) prevalence has remained high with an estimated annual number of infections of 130 million worldwide in 2012. ${ }^{1}$ Chlamydia disproportionately affects women of reproductive age with a prevalence of 4.0\% compared with $2.8 \%$ among men. ${ }^{1}$ Studies indicate that $10 \%-30 \%$ of women experience one or more chlamydia episodes. ${ }^{23}$ With up to $70 \%$ of infections being asymptomatic, many women are left untreated and are prone to chlamydia-related sequelae ${ }^{4}$ such as pelvic inflammatory disease (PID), ectopic pregnancy and tubal factor infertility (TFI). ${ }^{5}$

Proportions of PID following chlamydia were found between $3.0 \%$ and $30.0 \%,{ }^{6-10}$ for ectopic pregnancy between $0.2 \%$ and $2.7 \%,^{7-11}$ and for TFI between $0.1 \%$ and $6.0 \%{ }^{6712}$ Questions remain concerning true risks of sequelae due to study limitations, such as small sample size or limited follow-up time, ${ }^{13}$ unavoidable misclassification of chlamydia status as it is primarily based on incident (nucleic acid amplification test (NAAT)) tests, ${ }^{9}$ or lack of information on potential confounders such as sexual risk behaviour, demographic data and lifestyle factors in cohort studies based on large medical databases. ${ }^{10}$ These limitations need to be addressed to determine true risks of chlamydia-related sequelae.

The only way to interrupt the course of a chlamydia infection is to test and treat women at risk for chlamydia. However, there is no scientific evidence that screening decreases prevalence. ${ }^{2} 1415$ It might be more effective to move towards targeted screening of women at highest risk for developing sequelae since the clinical course of chlamydia differs greatly between individuals and depends on pathogen factors, environmental factors and host factors. ${ }^{9}$ 16-19 To study chlamydia-related sequelae and risk factors, the Netherlands Chlamydia Cohort Study (NECCST) was initiated in 2015. NECCST is an ongoing cohort study among Dutch women of reproductive age and a follow-up study of the 
2008-2011 Chlamydia Screening Implementation programme (CSI) in which all women were tested for chlamydia. ${ }^{20}$ We estimated risks of PID, ectopic pregnancy and TFI up to 8 years after chlamydia infection in women taking into account sexual risk behaviour, demographics and lifestyle factors. To identify women with high risk for sequelae, potential risk factors were evaluated.

\section{METHODS}

\section{Study design and participants}

NECCST is a cohort of women of reproductive age to be followed until 2022. The design has been described previously. ${ }^{20}$ Briefly, women between 16 and 29 years participated in 20082011 in the CSI. ${ }^{14}$ CSI participants were tested for chlamydia via PCR test $\geq 1$ with a maximum of four times in a 4 -year period. In case of chlamydia-positive PCR outcomes, participants were referred to the general practitioner or STI clinic for treatment. CSI women were traced in municipal registers and invited for participation in NECCST (online supplementary figure S1).

\section{Procedures}

In 2015-2016, women were invited and informed by regular mail and email. The first NECCST data collection moment included an electronic questionnaire followed by a test kit for self-collection of blood via a finger-prick for chlamydia $\operatorname{IgG}$ analyses as a marker for previous infection. The initial NECCST questionnaire retrospectively inquired about previous chlamydia infections, PID, ectopic pregnancy and TFI. Additionally, the NECCST questionnaire addressed demographic factors, sexual behaviour, other STIs, contraceptive use and health characteristics. For all events, timing in calendar year was asked. This was done to reconstruct a timeline for time-to-event analysis. Subsequently, CSI data about chlamydia PCR results, self-reported chlamydia infections and age of sexual debut acquired via the CSI-questionnaires were merged with NECCST data.

Chlamydia IgG antibodies were determined in self-collected capillary blood samples, collected in tubes (BD Microtainer SST, USA) and returned to the laboratory via mail. Serum samples were stored at $-20^{\circ} \mathrm{C}$ until thawed for ELISA with a sensitivity of $71 \%$ and specificity of $97 \%$ (Medac CT IgG ELISA plus, Wedel, Germany). ${ }^{21} 22$

\section{Definitions and outcomes}

Chlamydia positivity was defined as a positive PCR test outcome in the CSI study (CSI-PCR +), and/or the presence of chlamydia IgG in serum and/or a self-reported chlamydia infection. From the year of first chlamydia infection onwards, a woman was classified as chlamydia positive and remained positive. If year of first infection was unknown, that is, in women with a positive chlamydia IgG result only, multiple imputations were done for the Cox model analyses using truncated regression with 15 simulation datasets ${ }^{23}$ to estimate time of first chlamydia infection following sexual debut based on available data from women with a known year of first infection. Women were classified as chlamydia negative when they were CSI-PCR negative, had no chlamydia antibodies and did not report a previous infection.

The primary outcomes were PID, ectopic pregnancy and TFI: 1. PID was defined as a self-reported episode of inflammation of the ovaries, uterus and/or fallopian tubes, diagnosed by a medical professional. To obtain more information, women were asked whether the diagnosis was based on reported symptoms, physical examination, laboratory testing (either blood or vaginal swab examination), laparoscopy or whether this was unknown to them. Furthermore, we asked if women had been admitted to the hospital for the PID episode.

2. Ectopic pregnancy was defined as a self-reported extrauterine pregnancy. Women who were/had been pregnant were asked if they had experienced an ectopic pregnancy, and if so, the number and year of ectopic pregnancies.

3. TFI was defined as self-reported infertility caused by abnormalities of the tubes (possibly combined with other causes of infertility), diagnosed by a medical professional. All women who reported infertility were asked which fertility examinations had been conducted, that is, chlamydia antibody testing, hysterosalpingography, contrast ultrasonography, laparoscopy or 'none of the above'. Although self-reported TFI was found a reliable measure of TFI in the Netherlands, ${ }^{24}$ we validated a subset of self-reported TFI cases. Substantial agreement (Kappa value 0.73 ) between self-reported TFI and medical registers was found in the first NECCST round.

Exposure time in time-to-event analyses was defined as the total number of person-years, starting from when a woman became sexually active (or minimum age defined at 12 years for earlier reports). Exposure time ended at last data collection point or when a specific outcome event occurred.

\section{Statistical analyses}

Incidence rates (calculated as the number of new outcomes divided by total person-years at risk) of primary study endpoints PID, ectopic pregnancy and TFI were described.

The association between chlamydia status and each of the primary study endpoints was retrospectively assessed through Kaplan-Meier curves and univariable and multivariable Cox proportional-hazards regression models. Risks were expressed in HRs. Chlamydia status was included as a time-dependent variable. The following variables were considered potential confounders and included in the model if there was a $\geq 10 \%$ change in the regression coefficient: age (time-varying), gonorrhoea infection, migration background (Western vs non-Western, based on parental country of birth), educational level low/middle versus high (ie, low/medium: no education, primary education only, lower general secondary education, and vocational education; high level: all other education levels), lifetime sex partners, age of sexual debut, condom use with casual partner, smoking behaviour and intrauterine device (IUD) insertion. The proportional-hazard assumption was checked using log-log plots and testing Schoenfeld residuals.

To be able to identify chlamydia-positive women with a high risk for sequelae, risk factors for PID and TFI were determined by Cox regression models. Included risk factors were demographics, sexual risk behaviour, contraceptive use, chlamydia infection variables and serology (positive/negative outcome). All variables were assessed via univariable analysis and those associated $(p<0.10)$ with the outcome were entered via backward stepwise selection into the multivariable model.

To establish how closely risk factors were associated with the outcomes regardless of chlamydia status, factors were univariably tested with each outcome.

Multiple imputations and analyses were performed in STATA (V.14; StataCorp, College Station, Texas, USA).

\section{Sensitivity analyses}

First, we repeated analyses without multiple imputations for women with an unknown year of chlamydia infection. Second, analyses were repeated including only women with CSI-PCR-positive test results because CSI-PCR results have high sensitivity 
and specificity and no recall bias. Third, repeated analyses for ectopic pregnancy and TFI were restricted to women who had been pregnant at least once or who had ever tried to become pregnant. Fourth, due to residual uncertainties in PID diagnosis, we repeated analyses for PID including only hospitalised cases and for PID cases that occurred later than year of first chlamydia infection. This was not indicated for ectopic pregnancy and TFI since for both only one case occurred in the same year as the first chlamydia infection.

\section{RESULTS}

Between November 2015 and August 2016, 13 498/14 865 (91.9\%) of eligible CSI women were able to be retraced and invited for NECCST. Moreover, 5704 (42.3\%) women were enrolled and completed the initial questionnaire (online supplementary figure S1). In total, $1682(29.5 \%$, 95\% CI 28.3 to 30.7 ) women were ever chlamydia positive and 4022 $(70.5 \%, 95 \%$ CI 0.69 to 0.72$)$ had remained chlamydia negative. Chlamydia positivity was based on 1469 (87.3\%) self-reported infections, of which $341(23.2 \%)$ also tested PCR positive during CSI, and 360 (24.5\%) also tested positive for chlamydia IgG. Chlamydia antibody testing was done in 3670 (65\%) women of whom $15.5 \%$ (95\% CI 14.3 to 16.7 ) was positive. The remaining chlamydia positives $(n=213)$ only had a positive chlamydia IgG test $(n=208)$, were only PCR positive in CSI $(n=4)$ or were both chlamydia IgG and PCR positive $(n=1)$ but did not report this (online supplementary figure S2). Multiple imputations to impute time for first chlamydia were used for 226/1682 (13.4\%) chlamydia-positive women. These women were slightly older (31.6 vs 30.8 years) and had less lifetime partners and more condom use compared with chlamydia positives with known date of infection. Mean age at start of exposure time (sexual debut) was 16.9 (SD 2.4) years and mean exposure time was 14.2 (SD 3.9) years. Total exposure time was 86610 person-years (py). Characteristics of study participants are presented in table 1 .

\section{Pelvic inflammatory disease}

Women with an episode of PID before exposure time were excluded $(\mathrm{n}=6)$. In total, $159(2.8 \%, 95 \%$ CI 2.4 to 3.2$)$ women reported at least one episode of PID, of which 57 (35.9\%) were admitted to hospital. Of 33 (20.8\%) women with $>1$ reported PID episode, $45.5 \%$ were admitted to hospital at least once. The incidence rate of PID was 1.8 per 1000 py (95\% CI 1.6 to 2.2). Chlamydia positives had a higher incidence rate of 4.4 episodes of PID per 1000 py (95\% CI 3.3 to 5.7 ) compared with 1.4 per 1000 py (95\% CI 1.1 to 1.7 ) for chlamydia negatives. Incidence rates among the different chlamydia parameters, CSI-PCR +, chlamydia antibody presence or self-reported infections, were 4.5 (95\% CI 2.6 to 7.9 ), $5.2(95 \%$ CI 2.9 to 9.1$)$ and 4.1 (95\% CI 2.8 to 5.8) per 1000 py, respectively. In multivariable analysis, adjusted for age and educational level, chlamydia positivity remained strongly associated with PID (adjusted HR (aHR) 2.22, 95\% CI 1.57 to 3.13) (table 2 and figure 1).

\section{Ectopic pregnancy}

In total, $2633(46.2 \%)$ women were pregnant at least once or tried to become pregnant. Fifty-two $(0.9 \%, 95 \%$ CI 0.7 to 1.2$)$ women reported one or more ectopic pregnancies. The incidence rate of ectopic pregnancy was 0.6 per 1000 py $(95 \%$ CI 0.5 to 0.8 ). Ectopic pregnancy rates were not different between chlamydia positives ( 0.8 per 1000 py, $95 \%$ CI 0.4 to 1.5$)$ and
Table 1 Characteristics of the study population by chlamydia status at start of NECCST

\begin{tabular}{|c|c|c|c|c|}
\hline & $\begin{array}{l}\text { Overall } \\
\mathrm{n}(\%)\end{array}$ & $\begin{array}{l}\text { Chlamydia } \\
\text { negative } \\
\text { n (\%) }\end{array}$ & $\begin{array}{l}\text { Chlamydia } \\
\text { positive } \\
\text { n (\%) }\end{array}$ & $P$ value \\
\hline & $5704(100 \%)$ & $4022(70.5 \%)$ & $1682(29.5 \%)$ & \\
\hline Age (years) mean (SD) & $31.1(3.8)$ & $31.2(3.8)$ & $30.9(3.8)$ & $0.003^{*}$ \\
\hline $\begin{array}{l}\text { Chlamydia positivity in CSI- } \\
\text { PCR } n(\%)\end{array}$ & $908(15.9)$ & & $908(54.0)$ & \\
\hline Gonorrhoea positivity n (\%) & $112(2.0)$ & $32(0.8)$ & $80(4.8)$ & $<0.001^{*}$ \\
\hline Migration background & & & & $<0.001^{*}$ \\
\hline Western & $4565(80.0)$ & $3382(84.1)$ & $1183(70.3)$ & \\
\hline Non-Western & $869(15.2)$ & $464(11.5)$ & $405(24.1)$ & \\
\hline Unknown & $270(4.7)$ & $176(4.4)$ & $94(5.6)$ & \\
\hline Educational levelt‡ & & & & $<0.001^{*}$ \\
\hline Low/middle & $1170(20.5)$ & $661(16.5)$ & $509(30.3)$ & \\
\hline High & $4529(79.5)$ & $3358(83.6)$ & $1171(69.7)$ & \\
\hline $\begin{array}{l}\text { Age at sexual debut mean } \\
\text { (SD) }\end{array}$ & $16.9(2.4)$ & $17.2(2.4)$ & $16.4(2.1)$ & $<0.001 *$ \\
\hline Lifetime sex partners & & & & $<0.001^{*}$ \\
\hline$<6$ & $1797(31.5)$ & $1.499(37.3)$ & $298(17.7)$ & \\
\hline $6-12$ & $2033(35.6)$ & $1424(35.4)$ & $609(36.2)$ & \\
\hline$>12$ & $1874(32.9)$ & $1099(27.3)$ & $775(46.1)$ & \\
\hline $\begin{array}{l}\text { Condom use with casual } \\
\text { partners§ }\end{array}$ & & & & $<0.001^{*}$ \\
\hline Never/not often & $340(6.0)$ & $243(6.1)$ & $97(5.8)$ & \\
\hline Sometimes & $1807(31.7)$ & $1100(27.4)$ & $707(42.1)$ & \\
\hline Always/mostly & $2579(45.9)$ & $1890(47.1)$ & $720(42.9)$ & \\
\hline No casual partners & $936(16.4)$ & $780(19.4)$ & $156(9.3)$ & \\
\hline Use of IUD & & & & 0.230 \\
\hline Never & $3672(64.4)$ & $2609(64.9)$ & $1063(63.2)$ & \\
\hline At least once & $2032(35.6)$ & $1413(35.1)$ & $619(36.8)$ & \\
\hline Smoking & & & & $<0.001^{*}$ \\
\hline Never & $2264(39.7)$ & $1724(42.9)$ & $540(32.1)$ & \\
\hline Sometimes/in the past & $2843(49.8)$ & $1960(48.7)$ & $883(52.5)$ & \\
\hline Daily & $597(10.5)$ & $338(8.4)$ & $259(15.4)$ & \\
\hline No of chlamydia tests & & & & $<0.001^{*}$ \\
\hline 1 & $1042(18.3)$ & $931(23.2)$ & $111(6.6)$ & \\
\hline 2 & $1539(27.0)$ & $1222(30.4)$ & $317(18.9)$ & \\
\hline 3 & $1485(26.0)$ & $1032(25.7)$ & $453(26.9)$ & \\
\hline$>3$ & $1638(28.7)$ & $837(20.8)$ & $801(47.6)$ & \\
\hline \multicolumn{5}{|l|}{ Chlamydia symptoms $\uparrow$} \\
\hline No & & - & $952(56.6)$ & \\
\hline Yes & & - & $730(43.4)$ & \\
\hline \multicolumn{5}{|l|}{ No of chlamydia infections** } \\
\hline 1 & & - & $1325(78.8)$ & \\
\hline 2 & & - & $291(17.3)$ & \\
\hline 3 or more & & - & $66(3.9)$ & \\
\hline \multicolumn{5}{|l|}{ Age at first chlamydia infection } \\
\hline$<20$ & & - & $305(20.8)$ & \\
\hline $20-24$ & & - & $640(43.7)$ & \\
\hline$>24$ & & - & $519(35.5)$ & \\
\hline \multicolumn{5}{|c|}{ Chlamydia IgG antibodies (serology) } \\
\hline No & $3101(54.4)$ & $2572(64.0)$ & $529(31.5)$ & \\
\hline Yes & $569(10.0)$ & - & $569(33.8)$ & \\
\hline Unknown & $2034(35.7)$ & $1450(36.0)$ & $584(34.7)$ & \\
\hline
\end{tabular}

Chlamydia positive was defined as a positive PCR-test outcome in the CSI study (CSI-PCR), and/or the presence of chlamydia lgG and/or a self-reported chlamydia infection.

*Statistically significant, $p<0.05$

* Statistically significant, $p<0.05$. education and vocational education; high level of education: all other education levels. Chlamydia symptoms were vaginal discharge, abdominal pain or pain during intercourse and/or intermittent vaginal bleeding. ¥At start of NECCST and based on 5699 observations, 5 missing. \#At start of NECCST and based on 5699 o
§Based on 5693 observations, 11 missing. §Based on 5693 observations, 11 missing.
qbased on 1665 observations, 15 missing values.

**Based on 1639 observations, 43 missing.

CSI, Chlamydia Screening Implementation; IUD, intrauterine device; NECCST, Netherlands Chlamydia Cohort Study.

chlamydia negatives ( 0.6 per 1000 py, $95 \%$ CI 0.4 to 0.8 ), nor in multivariable analysis after adjustment for age, educational level, number of lifetime partners and migration background 
Table 2 Association between chlamydia positivity and pelvic inflammatory disease, ectopic pregnancy and tubal factor infertility in women participating in NECCST

\begin{tabular}{|c|c|c|c|c|c|c|c|c|}
\hline & \multirow{2}{*}{$\begin{array}{l}\text { Cases } \\
\text { N§ }\end{array}$} & \multirow{2}{*}{$\begin{array}{l}\text { Time } \\
\text { Person-years } \emptyset\end{array}$} & \multicolumn{3}{|c|}{ Crude HR } & \multicolumn{3}{|c|}{ Adjusted HR*†‡ } \\
\hline & & & HR & $95 \% \mathrm{Cl}$ & $P$ value & aHR & $95 \% \mathrm{Cl}$ & $P$ value \\
\hline \multicolumn{9}{|c|}{ Pelvic inflammatory disease } \\
\hline Chlamydia negative & 100 & 71029 & 1 & & & 1 & & \\
\hline Chlamydia positive & 59 & 14320 & 2.60 & 1.85 to 3.66 & $<0.0001$ & 2.22 & 1.57 to 3.13 & $<0.0001$ \\
\hline \multicolumn{9}{|l|}{ Ectopic pregnancy } \\
\hline Chlamydia negative & 41 & 71412 & 1 & & & 1 & & \\
\hline Chlamydia positive & 11 & 14684 & 0.99 & 0.49 to 1.97 & & 0.80 & 0.39 to 1.63 & 0.5335 \\
\hline \multicolumn{9}{|l|}{ Tubal factor infertility } \\
\hline Chlamydia negative & 15 & 71523 & 1 & & & 1 & & \\
\hline Chlamydia positive & 18 & 14699 & 4.27 & 2.08 to 8.77 & 0.0001 & 4.22 & 2.05 to 8.69 & 0.0001 \\
\hline
\end{tabular}

Chlamydia positive was defined as a positive PCR-test outcome in the CSI study (CSI-PCR), and/or the presence of chlamydia IgG and/or a self-reported chlamydia infection. For these analyses, multiple imputations were used to estimate time of first chlamydia infection in women without a known first year of chlamydia infection.

*PID model adjusted for age (time-varying) and educational level.

tEctopic pregnancy model adjusted for age (time-varying), educational level, number of lifetime partners and migration background.

¥Tubal factor infertility model adjusted for age (time-varying).

$\S$ Median cases of 15 multiple imputation datasets.

IEstimated from 15 multiple imputation datasets.

aHR, adjusted HR; CSI, Chlamydia Screening Implementation; NECCST, Netherlands Chlamydia Cohort Study.

(aHR 0.80, 95\% CI 0.39 to 1.63 ) (table 2, figure 1). Incidence rates among the different chlamydia parameters, CSI-PCR+, chlamydia antibody presence or self-reported infections, were 0.4 (95\% CI 0.1 to 2.6$), 0.4$ (95\% CI 0.1 to 3.0$)$ and $1.1(95 \%$ CI 0.5 to 2.1$)$ per 1000 py, respectively.

\section{Tubal factor infertility}

In total, $33(0.6 \%, 95 \%$ CI 0.4 to 0.8$)$ women reported to be diagnosed with TFI. The incidence rate was 0.4 per 1000 person-years (95\% CI 0.3 to 0.5 ). Among chlamydia positives, the incidence rate of TFI was considerably higher compared with chlamydia negatives: 1.3 per 1000 py (95\% CI 0.8 to 2.1 ) and 0.2 per 1000 py (95\% CI 0.1 to 0.4 ), respectively. Incidence rates among the different chlamydia parameters, CSI-PCR+, chlamydia antibody presence or self-reported infections, were 1.1 (95\% CI 0.4 to 3.4 ), 0.8 (95\% CI 0.2 to 3.4) and 1.4 (95\% CI 0.8 to 2.6) per 1000 py, respectively. Chlamydia positivity was highly associated with TFI: aHR 4.22 (95\% CI 2.05 to 8.69 ) (table 2 and figure 1). The model was adjusted for age.

Sensitivity analyses on all three outcomes (PID, ectopic pregnancy, TFI) using data restrictions as indicated in methodology section yielded mostly similar results (figure 2).

\section{Risk factors for long-term sequelae among chlamydia- positive women}

In multivariable analyses, having had chlamydia symptoms and IUD insertion were associated with PID (online supplementary table 1). For TFI, a younger age at first chlamydia infection ( $<20$ years) was the only risk factor in univariable analyses. For both PID and TFI, we were unable to determine an increased risk for women with an unnoticed chlamydia infection (chlamydia IgG-positive only) compared with women with a known and presumably treated infection.

Associations between risk factors and outcomes regardless of chlamydia status can be found in online supplementary table S2.

\section{DISCUSSION}

We estimated risks of PID, ectopic pregnancy and TFI in a unique cohort of over 5500 women with up to 8 years of follow-up. Chlamydia positivity was strongly associated with PID, as shown by a twofold higher risk, and with TFI, by a fourfold higher risk. Incidence rates and risks on sequelae were comparable among all chlamydia positives (either PCR positive, antibody positive or self-reported). Among chlamydia-positive women, PID was found to be more common in women who had symptoms during infection. For TFI among chlamydia-positive women, young age $(<20)$ at first chlamydia infection was the strongest risk factor. We did not find an association between chlamydia positivity and ectopic pregnancy. Although differences in risk between chlamydia-positive and chlamydia-negative women were considerable and highly significant, the cumulative incidence for any of the sequelae studied was low $(<5 \%)$.

This cohort study yielded an exhaustive dataset in which chlamydia-related sequelae were determined. First, over 5500 women were followed since 2008 with multiple data collection points. Second, we adopted a thorough approach in determining previous chlamydia infections using three outcome measures: CSI-PCR, serology and self-reporting. Thereby increasing the true proportion of chlamydia-positive women, however, we cannot completely exclude infections in the negative group due to moderate sensitivity of the chlamydia IgG ELISA test. Third, we were able to adjust the relation between chlamydia and its sequelae for multiple confounders, that is, sexual risk behaviour, previous pregnancies and pregnancy wishes, demographics and lifestyle factors. Therefore, our results may approach true risk estimates by reducing some important uncertainties. ${ }^{5}$

This study had several limitations. First, we used self-reported data on PID, ectopic pregnancy and TFI and in part on chlamydia infections, which are subject to recall bias. In addition, PID diagnoses are imprecise since a non-invasive gold standard test is not available. ${ }^{25}$ Both over-reporting and diagnostic bias towards chlamydia-positive women could have taken place, ${ }^{5}$ possibly leading to an overestimation of the risk 


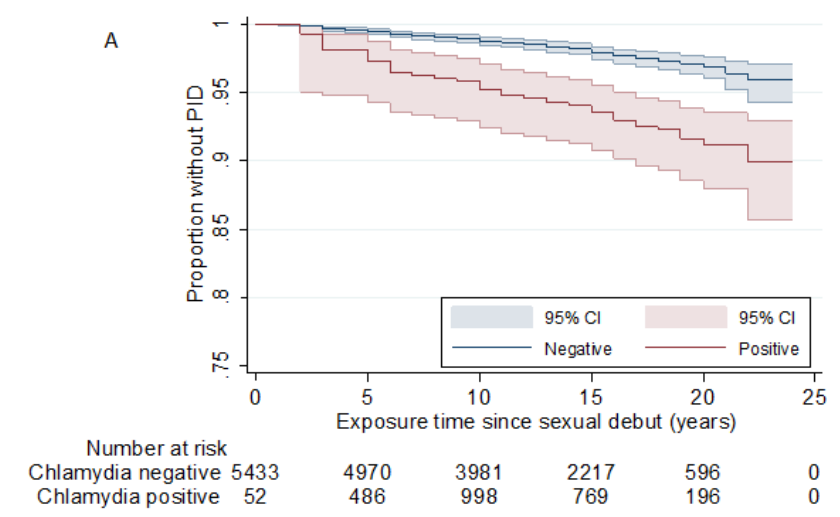

B

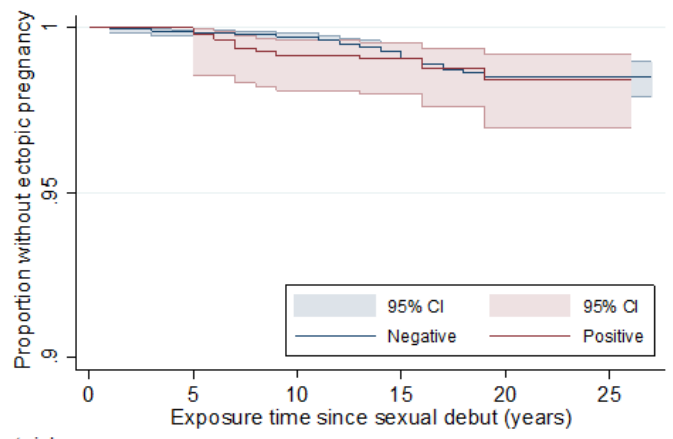

Number at risk

$\begin{array}{ccccccc}\text { Number at risk } & & & & \\ \text { Chlamydia negative } 5433 & 4983 & 4011 & 2241 & 601 & 9 \\ \text { Chlamydia positive } & 52 & 492 & 1015 & 799 & 211 & 9\end{array}$

$\mathrm{C}$

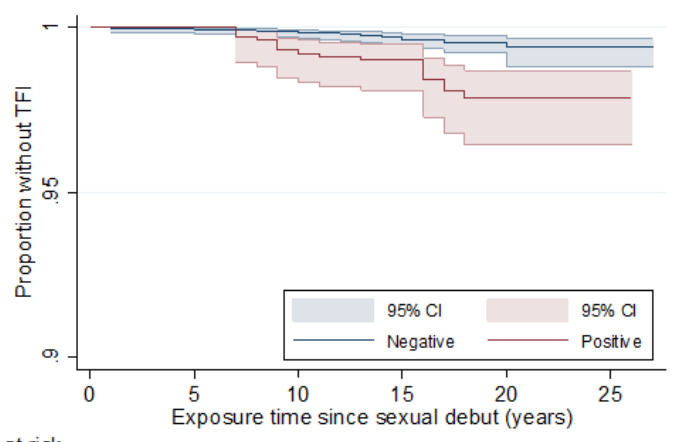

Number at risk

$\begin{array}{ccccccc}\text { Chlamydia negative } 5433 & 4987 & 4015 & 2249 & 607 & 9 \\ \text { Chlamydia positive } & 52 & 492 & 1014 & 798 & 215 & 8\end{array}$

Figure 1 Kaplan-Meier plots of time (years) by chlamydia status since sexual debut. (A) Pelvic inflammatory disease (PID). (B) Ectopic pregnancy. (C) Tubal factor infertility (TFI). Chlamydia positive was defined as a positive PCR-test outcome in the CSI study (CSI-PCR), and/ or the presence of chlamydia IgG and/or a self-reported chlamydia infection. CSI, Chlamydia Screening Implementation.

among chlamydia-positive women. TFI was based on self-reports, but partly validated in medical registers, showing good agreement. Second, because the NECCST cohort is based on a sample of the CSI study, we have a non-representative sample which decreased generalisability of the results. Third, timing of events was defined in years. As PID sometimes occurred in the same year as the first chlamydia infection, it cannot be ruled out that some PID cases might have occurred before the first chlamydia infection. Sensitivity analyses excluding PID cases that occurred in the same year as the chlamydia infection still yielded an increased risk for chlamydia-positive women, though not significant. Thus, while the largest risk for PID following a chlamydia infection was in the year of infection, the majority of PIDs occurred thereafter. Lastly, to draw conclusions on a possible association between chlamydia and ectopic pregnancy, which remains inconclusive also in previous studies, ${ }^{926}$ longer follow-up of NECCST will be needed. ${ }^{20}$

Incidence rates of PID and TFI among chlamydia-positive women found in our study (4.4/1000 and 1.1/1000 py, respectively) were comparable with results from two Danish studies and an Australian population-based cohort study. However, our incidence rate for ectopic pregnancy was lower: 0.8/1000 py. These studies found incidence rates of 4.0-8.0/1000 py for PID, 2.8/1000 py for ectopic pregnancy and $0.7 / 1000$ py for TFI and reported cumulative incidences of $2 \%-3 \%$, 2\%-3\% and $0.6 \%$, respectively. ${ }^{9} 1027$ For PID and TFI, the Danish and Australian studies found smaller differences in risk between chlamydia positives and negatives than we did: for PID 1.3-1.8 and for TFI $1.3(9,10,27)$ compared with our HRs of 2.2 and 4.2, respectively. A different comparison was made in these studies because their definition of chlamydia negative was based on negative test (NAAT) results, excluding non-tested women, who were at lower risk than women who tested negative.

We want to be able to identify chlamydia-positive women with the highest risk of complications. Identified risk factors can help develop chlamydia-specific prevention methods. In chlamydia-negative women, it is more likely that the cause of complications is due to causes other than chlamydia. The strongest risk factors for PID among chlamydia-positive women were chlamydia symptoms and insertion of an IUD. However, women with an IUD might be more focused on abdominal pain and visit a doctor earlier compared with women without an IUD (diagnostic bias). ${ }^{28}$ Thereby, only part of PID, but also ectopic pregnancy and TFI, is caused by chlamydia; estimated population excess fractions are between $20 \%$ and $30 \%$ for PID, and around 5\% for ectopic pregnancy and $30 \%$ for TFI. $^{7}$

This study adds to the evidence that chlamydia increases the risk for PID and TFI in women even if the infection was treated, ${ }^{29}$ but also showed that incidence rates were small. However, alertness for PID in years after infection remains warranted among healthcare providers, especially in women who experienced chlamydia-related symptoms during infection. Though chlamydia is an important causative agent for TFI (ie, fourfold increased risk) during the 8-year follow-up time of our cohort, TFI was only found in $1 \%$ of women following chlamydia infection, in accordance with modelling studies. ${ }^{12}$ It is important to identify that $1 \%$ of women had high risk. The following step in NECCST is to assess single-nucleotide polymorphisms (SNPs) associations.

The present results underscore the need for effective prevention of reproductive tract complications following chlamydia infection. Given the small cumulative risks and the lack of evidence that chlamydia screening is effective in decreasing prevalence, it might be more effective to focus on women at highest risk for complications rather than promoting widespread chlamydia screening. ${ }^{30}$ 


\section{Forest plot sensitivity analyses}

$\mathrm{HR}(95 \% \mathrm{Cl})$

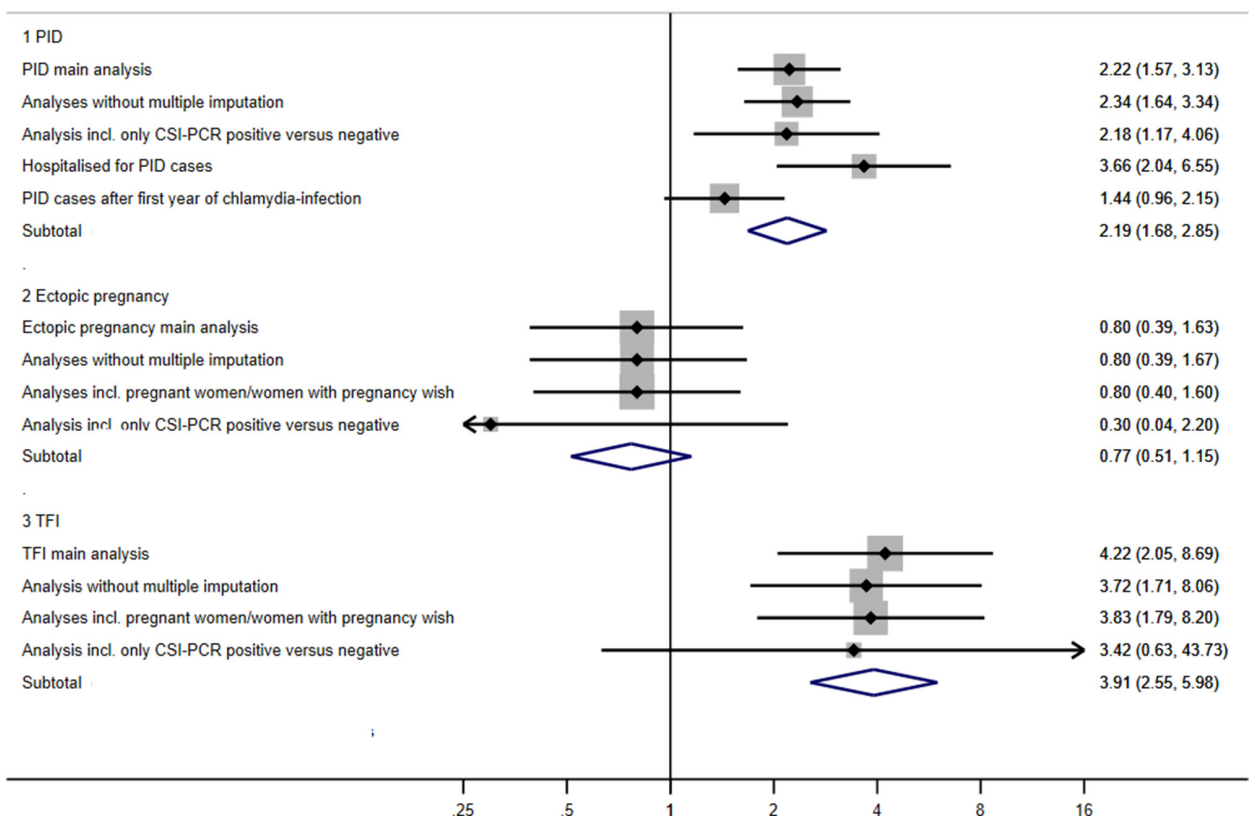

Figure 2 Forest plot of various sensitivity analyses of the risk for pelvic inflammatory disease (PID), ectopic pregnancy and tubal factor infertility (TFI) between chlamydia-positive and chlamydia-negative women in HRs and 95\% Cl. CSI, Chlamydia Screening Implementation.

\section{Key messages}

- Estimating risks of Chlamydia trachomatis-related sequelae is difficult due to the asymptomatic nature of infection, other causes involved and time duration between infection and complications.

- In this long-term observational cohort study of women of reproductive age, chlamydia was measured using nucleic acid amplification test outcomes, serology and self-reported infections with $30 \%$ chlamydia positivity.

- Women who had chlamydia were at higher risk for pelvic inflammatory disease (twofold) and tubal factor infertility (fourfold), but not for ectopic pregnancy. Incidences rates, however, were small.

- Targeting women at highest risk for complications might be a more effective strategy to prevent Chlamydia-related complications than widespread Chlamydia screening.

\footnotetext{
Author affiliations

Epidemiology and Surveillance Unit, Centre for Infectious Disease Control, National Institute for Public Health and the Environment, Bilthoven, The Netherlands 'Laboratory of Immunogenetics, Department of Medical Microbiology and Infection Control, VU University Medical Center, Amsterdam, The Netherlands

${ }^{3}$ Department of General Practice, Division Clinical Methods and Public Health, Academic Medical Center, Amsterdam, The Netherlands

${ }^{4}$ STI AIDS Netherlands (SOA AIDS Nederland), Amsterdam, The Netherlands ${ }^{5}$ Department of Sexual Health, Infectious Diseases and Environmental Health, South Limburg Public Health Service (GGD South Limburg), Geleen, The Netherlands ${ }^{6}$ Department of Medical Microbiology, Care and Public Health Research Institute (CAPHRI), Maastricht University Medical Centre (MUMC+), Maastricht, The Netherlands

${ }^{7}$ Department Infectious Disease Control, Municipal Public Health Service RotterdamRijnmond (GGD Rotterdam), Rotterdam, The Netherlands

${ }^{8}$ Department of Public Health, Erasmus MC — University Medical Center Rotterdam, Rotterdam, The Netherlands
}

${ }^{9}$ STI Outpatient Clinic, Public Health Service of Amsterdam (GGD Amsterdam), Amsterdam, The Netherlands

${ }^{10}$ Department of Genetics and Cell Biology, Research School GROW (School for Oncology and Developmental Biology), Faculty of Health, Medicine and Life Sciences, Institute for Public Health Genomics (IPHG), University of Maastricht, Maastricht, The Netherlands

${ }^{11}$ Julius Center for Health Sciences and Primary Care, University Medical Center Utrecht, Utrecht, The Netherlands

${ }^{12}$ Department of Public Health, Institute of Tropical Medicine, Antwerp, Belgium

\section{Handling editor Jane S Hocking}

Acknowledgements The authors thank J Pleijster, senior research technician, for her work on the chlamydia lgG antibody assays. We acknowledge the editing and language services of Mr K Jamdagni.

Contributors All authors developed the study design. BMH collected the data for NECCST and performed among others the laboratory analyses. SAM coordinated the laboratory analyses. BMH led on the statistical analyses with oversight of IVFvdB and BHBvB. The data interpretation was done by BMH, IVFvdB, BHBvB and SAM and discussed with JEAMvB, MABvdS, JAL, HMG, CJPAH and NHTMD-M. All authors contributed to drafting and revision of the paper and all authors approved the final manuscript.

Funding This work was supported by the Netherlands Organisation for Health Research and Development (ZonMW Netherlands, a governmental organisation grant (registration no. 50-53000-98-103)) and Research Funding from the Ministry of Health, Welfare and Sports to the Centre of Infectious Disease Control.

Disclaimer The funders had no role in study design, data collection and analysis, interpretation of data, decision to publish or preparation of the manuscript.

Competing interests None declared.

Patient consent for publication Obtained.

Ethics approval This study was approved by the Medical Ethical Committee Noord-Holland, Alkmaar, the Netherlands (NL 51553.094.14/M014-042).

Provenance and peer review Not commissioned; externally peer reviewed.

Data sharing statement On request, (anonymised) data and available biological material can be provided for research related to STI, after approval by an advisory committee.

Open access This is an open access article distributed in accordance with the Creative Commons Attribution Non Commercial (CC BY-NC 4.0) license, which 
permits others to distribute, remix, adapt, build upon this work non-commercially, and license their derivative works on different terms, provided the original work is properly cited, appropriate credit is given, any changes made indicated, and the use is non-commercial. See: http://creativecommons.org/licenses/by-nc/4.0/.

\section{REFERENCES}

1 Newman L, Rowley J, Vander Hoorn S, et al. Global estimates of the prevalence and incidence of four curable sexually transmitted infections in 2012 based on systematic review and global reporting. PLoS One 2015;10:e0143304.

2 Woodhall SC, Wills GS, Horner PJ, et al. Chlamydia trachomatis Pgp3 antibody population seroprevalence before and during an era of widespread opportunistic chlamydia screening in England (1994-2012). Plos One 2017;12:e0152810.

3 van Aar F, de Moraes M, Morré SA, et al. Chlamydia trachomatis IgG seroprevalence in the general population of the Netherlands in 1996 and in 2007: differential changes by gender and age. Sex Transm Infect 2014;90:434-40.

4 Low N, McCarthy A, Macleod J, et al. Epidemiological, social, diagnostic and economic evaluation of population screening for genital chlamydial infection. Health Technol Assess 2007;11:1-165.

5 Haggerty CL, Gottlieb SL, Taylor BD, et al. Risk of sequelae after Chlamydia trachomatis genital infection in women. J Infect Dis 2010;201(S2):134-55.

6 Land JA, Van Bergen JE, Morré SA, et al. Epidemiology of Chlamydia trachomatis infection in women and the cost-effectiveness of screening. Hum Reprod Update 2010;16:189-204.

7 Price MJ, Ades AE, Soldan K, et al. The natural history of Chlamydia trachomatis infection in women: a multi-parameter evidence synthesis. Health Technol Assess 2016;20:1-250

8 van Valkengoed IG, Morré SA, van den Brule AJ, et al. Overestimation of complication rates in evaluations of Chlamydia trachomatis screening programmes-implications for cost-effectiveness analyses. Int J Epidemiol 2004;33:416-25.

9 Davies B, Turner KME, Frølund M, et al. Risk of reproductive complications following chlamydia testing: a population-based retrospective cohort study in Denmark. Lancet Infect Dis 2016;16:1057-64.

10 Low Net al. Incidence of severe reproductive tract complications associated with diagnosed genital chlamydial infection: the Uppsala Women's Cohort Study. Sexually Transmitted Infections 2006:82:212-8.

11 Bakken IJ, Skjeldestad FE, Lydersen S, et al. Births and ectopic pregnancies in a large cohort of women tested for Chlamydia trachomatis. Sex Transm Dis 2007:34:739-43.

12 Kavanagh K, Wallace LA, Robertson C, et al. Estimation of the risk of tubal factor infertility associated with genital chlamydial infection in women: a statistical modelling study. Int J Epidemio/ 2013;42:493-503.

13 Oakeshott P, Kerry S, Aghaizu A, et al. Randomised controlled trial of screening for Chlamydia trachomatis to prevent pelvic inflammatory disease: the POPI (prevention of pelvic infection) trial. BMJ 2010;340:c1642.

14 van den Broek IV, van Bergen JE, Brouwers EE, et al. Effectiveness of yearly, register based screening for chlamydia in the Netherlands: controlled trial with randomised stepped wedge implementation. BMJ 2012;345:e4316.
15 Low N, Redmond S, Uusküla A, et al. Screening for genital chlamydia infection. Cochrane Database Syst Rev 2016;9:CD010866.

16 Menon S, Timms P, Allan JA, et al. Human and pathogen factors associated with Chlamydia trachomatis-related infertility in women. Clin Microbiol Rev 2015;28:969-85.

17 Tiitinen A, Surcel HM, Halttunen M, et al. Chlamydia trachomatis and chlamydial heat shock protein 60-specific antibody and cell-mediated responses predict tubal factor infertility. Hum Reprod 2006;21:1533-8.

18 Morré SA, Karimi O, Ouburg S. Chlamydia trachomatis: identification of susceptibility markers for ocular and sexually transmitted infection by immunogenetics. FEMS Immunol Med Microbiol 2009;55:140-53.

19 Hay PE, Kerry SR, Normansell R, et al. Which sexually active young female students are most at risk of pelvic inflammatory disease? A prospective study. Sex Transm Infect 2016;92:63-6.

20 Hoenderboom BM, van Oeffelen AA, van Benthem BH, et al. The Netherlands Chlamydia cohort study (NECCST) protocol to assess the risk of late complications following Chlamydia trachomatis infection in women. BMC Infect Dis 2017:17:264.

21 Hoenderboom BM, van Ess EF, van den Broek IVF, et al. Chlamydia trachomatis antibody detection in home-collected blood samples for use in epidemiological studies. J Microbiol Methods 2018;144:164-7.

22 Morré SA, Munk C, Persson K, et al. Comparison of three commercially available peptide-based immunoglobulin $\mathrm{G}(\mathrm{lgG})$ and $\lg A$ assays to microimmunofluorescence assay for detection of Chlamydia trachomatis antibodies. J Clin Microbiol 2002:40:584-7.

23 Raghunathan TE, Lepkowski JM, Van Hoewyk J, et al. A multivariate technique for multiply imputing missing values using a sequence of regression models. Survey Methodology 2001;27(No. 1):85-95.

24 de Boer EJ, den Tonkelaar I, Burger CW, et al. Validity of self-reported causes of subfertility. Am J Epidemiol 2005;161:978-86.

25 Simms I, Warburton F, Weström L. Diagnosis of pelvic inflammatory disease: time for a rethink. Sex Transm Infect 2003;79:491-4.

26 Andersen $B$, Østergaard L, Puho E, et al. Ectopic pregnancies and reproductive capacity after Chlamydia trachomatis positive and negative test results: a historical follow-up study. Sex Transm Dis 2005;32:377-81.

27 Reekie J, Donovan B, Guy R, et al. Risk of pelvic inflammatory disease in relation to chlamydia and gonorrhea testing, repeat testing, and positivity: a population-based cohort study. Clinical Infectious Diseases 2018;66:437-43.

28 Hubacher D, Grimes DA, Gemzell-Danielsson K. Pitfalls of research linking the intrauterine device to pelvic inflammatory disease. Obstet Gynecol 2013;121:1091-8.

29 Dahlberg J, Hadad R, Elfving K, et al. Ten years transmission of the new variant of Chlamydia trachomatis in Sweden: prevalence of infections and associated complications. Sex Transm Infect 2018:94:100-104.

30 Unemo M, Bradshaw CS, Hocking JS, et al. Sexually transmitted infections: challenges ahead. Lancet Infect Dis 2017;17:e235-e279. 